\title{
BMJ Open Return to employment for working-aged adults after burn injury: a scoping review protocol
}

\author{
Akane Katsu (D) , , Zephanie Tyack (D) , ${ }^{3}$ Martin Mackey, ${ }^{4}$ James M Elliott,, 4 \\ Lynette Mackenzie ${ }^{1}$
}

To cite: Katsu A, Tyack $Z$, Mackey M, et al. Return to employment for workingaged adults after burn injury: a scoping review protocol. BMJ Open 2021;11:e044145. doi:10.1136/ bmjopen-2020-044145

- Prepublication history and supplemental material for this paper are available online. To view these files, please visit the journal online (http://dx.doi. org/10.1136/bmjopen-2020044145).

Received 24 August 2020 Revised 15 December 2020 Accepted 21 December 2020

Check for updates

(C) Author(s) (or their employer(s)) 2021. Re-use permitted under CC BY-NC. No commercial re-use. See rights and permissions. Published by BMJ.

For numbered affiliations see end of article.

Correspondence to Ms Akane Katsu; akat7413@uni.sydney.edu.au

\section{ABSTRACT}

Introduction Cutaneous burns can have a catastrophic effect on people's lives and may restrict opportunities for employment due to physical impairment and psychosocial deficits. Failure or delay in return to work can result in loss of income and support for the family unit. It can also negatively affect life role and identity and present difficulties with future opportunities. Current literature indicates multiple discrete influences on return to work as a result of burn injury but an understanding of how working-aged adults resume employment after burn injury is lacking. This scoping review will provide a comprehensive overview of the current literature by mapping and consolidating knowledge in this area of burn recovery and thus provide an informative basis for developing return-to-work programmes for survivors of burn injury.

Methods and analysis This scoping review protocol will follow the Arksey and 0'Malley's (2005) methodological framework. A comprehensive search strategy has been developed with subject expert librarians. These databases were used: OvidSP: Medline, Embase, PsycINF0, PubMed and Cochrane Central Register of Controlled Trials and EBSCOhost: CINAHL and Scopus. Reference lists of selected full text will be hand searched for additional literature. To enhance consistency and rigour, all reviewers will undertake a calibration exercise before paired reviewers independently screen all records using Rayyan. Full-text articles meeting the study inclusion criteria will be retrieved and examined. Extracted data will be analysed using the International Classification of Functioning, Disability and Health.

Ethics and dissemination Ethics approval is generally not required for scoping reviews. Findings of this scoping review will be reported in a peer-reviewed journal and presented at conferences.

\section{INTRODUCTION \\ Background}

Burn injury results in changes to health with physical, psychological, social and financial well-being. ${ }^{1}$ Consistent with International Classification of Functioning, Disability and Health (ICF), ${ }^{23}$ in the short term, burn injuries have the potential to result in physical impairment, activity limitations, participation restrictions and environmental barriers

\section{Strengths and limitations of this study}

This scoping review protocol will provide a comprehensive framework investigating the gaps in the current literature on returning to employment after burn injury for working-aged adults.

- This protocol follows the latest guidelines for conducting scoping reviews and intends to use the recently published Preferred Reporting Items for Systematic Reviews and Meta-Analyses Extension for Scoping Reviews.

- Researchers undertaking this scoping review represent clinical and research expertise from burns, return to work and trauma.

- The study will be inclusive of all types of assessments, interventions and outcomes of return to employment after burn injury to enable a broad overview of this aspect of burn recovery.

- This scoping review will be limited to Englishlanguage literature from high-income countries (World Bank's 2019 definition) and the results may not be applicable to burn injury populations from low-income and middle-income countries.

making return to employment difficult on a person-by-person basis. ${ }^{45}$ Physical impairments such as scarring, contractures, chronic pain and itch and thermoregulation problems can result in long-term disability post burn. ${ }^{6-9}$ Psychological issues resulting from disfigurement, anxiety, depression and post-traumatic stress disorders also contribute to the longterm disability experienced by people with burn injuries. ${ }^{8-12}$ These long-term healthrelated complications affect participation in activities of daily living and resumption of life roles. Work and employment are considered major life roles in which a person can be expected to participate in and contribute to their communities. ${ }^{2}$ The terms 'work' and 'employment' are used interchangeably in this paper and refer to paid work. These terms are not currently distinguished in the literature. 
Return to work as an outcome is an emerging factor for how recovery from burn injury is measured. ${ }^{13}$ Ten million disability adjusted life years are lost as a result of firerelated burn injuries globally each year and over $40 \%$ of these occur in those aged 15-59 years, who are of working age. ${ }^{14}$ In Australia, $65 \%$ of hospitalised burn patients are $15-64$ years of age, with the largest proportion (30\%) in the 25-44 age group. ${ }^{15}$ These adults are most likely to sustain full-thickness burn injuries as well as larger size full-thickness burn injuries compared with all other age groups. ${ }^{15}$ High-income countries such as Australia, the USA and the Netherlands have similar mean healthcare costs per burn patient, averaging about US $\$ 88218$ in total and US $\$ 4159$ per $1 \%$ total body surface area (TBSA). ${ }^{16}$ In 2015 , over $\$ A 112$ million was spent on healthcare costs for burn injuries in Australia. ${ }^{17}$ Healthcare costs do not represent the entirety of the economic burden of burn injuries with $90 \%$ of costs shouldered by patients and their families because of the loss of productive income and informal care needs. ${ }^{16}{ }^{18}$ Working-age adults are financially responsible for their dependants and are likely to be the most economically productive members of their communities. The ability to return to and effectively manage one's employment role at work is thus an important indicator of the recovery made by working-age adults as it demonstrates the reintegration into community and restoration of functional performance after burn injury. ${ }^{7}{ }^{13}$ 19-21 Rehabilitation for burn injury can assist individuals towards regaining their preinjury roles and survivors of burn injuries highly value rehabilitation that assist return to work. ${ }^{22}$

Studies have been undertaken to identify barriers to return to work for burn-injured patients, outcomes of intervention studies and how burn care centres address return to work in their clinical settings. ${ }^{11} 13192023-30$ Of these, at least four systematic reviews have investigated return to work after burn injury. ${ }^{13} 192029$ These reviews primarily investigated injury-related and personal factors affecting return to work. Per cent TBSA (\% TBSA) burn is the most commonly reported measure of burn severity, ${ }^{13} 1929$ followed by \%TBSA grafted and \%TBSA full-thickness burns. ${ }^{19}{ }^{29}$ Electrical burns result in more complex injury sequelae that delay return to work. ${ }^{20}$ Surprisingly, the presence of hand burns has not been conclusively associated with delayed return to employment. ${ }^{19}$ Length of stay is most likely a proxy marker for burn severity associated with delayed return to employment. ${ }^{19} 29$ People who have pre-existing medical and psychiatric conditions or who were unemployed prior to burn injury were less likely to re-engage in work post burn. ${ }^{10131929}$ Rates of return to work reported in the literature vary greatly-from $14 \%$ to $91 \%{ }^{29}$ with almost a third never returning to any form of work ${ }^{19}$ but few studies define what actually constitutes successful return to work.

The difficulty of and the lack of consistent definitions and outcome measures for reporting return to work in the burn literature have been highlighted in previous systematic reviews. ${ }^{13} 1929$ Clarification of work and employment for individuals may be helpful as work can represent different types of meaningful activities. While return to work has been used to describe both paid and unpaid work activities, ${ }^{19} 31$ employment is the means through which people are socially productive and refers to work done specifically to earn money. ${ }^{32}$ Without a clear definition of the meaning of work in this context, it will be difficult to achieve consistency in how return to work is quantified and outcomes measured. ${ }^{33}$

Although it is obvious that severe and catastrophic burns can result in complex recovery needs across the physical, psychological and social aspects of one's health and well-being, ${ }^{67910}$ disruption in health and well-being may also be experienced by those with less severe injuries. ${ }^{34}$ Currently, little is known about the return-to-work process for burns patients, which modifiable factors can be successfully used to assist return to work,${ }^{26}$ and what interventions are suitable at which time points in the burn recovery process. Previous reviews are limited by a focus on physical or psychological outcomes rather than work performance and environmental changes in the workplace. ${ }^{71927}$

\section{Aims and objectives}

The aim of this scoping review protocol is to map the body of literature informing what is known about workingaged adults with burn injuries returning to their previous employment. The objectives of this scoping review are to (a) provide a comprehensive overview of the current literature to consolidate our knowledge and (b) guide future research strategies measuring return to employment after burn injury.

\section{METHODS AND ANALYSIS}

A scoping review methodology is useful for examining a broad range of literature to explore complex, conceptual and emerging areas of research. ${ }^{35}$ This methodological approach is helpful for determining what the influences on return to employment are, the timeframes within which this should be achieved, the manner in which those injured return to employment and how such outcomes are accomplished. Studies on return to work for other population groups indicate the need to be comprehensive in assessment to look beyond injury factors and assess other aspects of recovery. ${ }^{36-39}$ There is scope to investigate how these factors have an impact on the ability to return to employment. For these reasons, a scoping review will be helpful in systematically mapping the research in this area to inform further study on return to employment after burn injury.

The scoping review methodology based on Arksey and O'Malley's ${ }^{40}$ framework modified by Levac et $a l^{41}$ and Peters $e t a t^{42}$ will be used to guide this review. Details of each stage of the scoping review process are described below.

\section{Stage 1: identifying the research question}

To ensure this scoping review would remain broadly focused on the topic area, we developed the primary 
research question: What is known and unknown about the body of evidence with respect to return to employment after burn injury?

We further identified subquestions to align our research question and objective:

- What are the barriers and benefits to return to employment experienced by people with burn injuries?

- What are the assessment tools or outcome measures used to qualify and/or quantify return to employment?

- What are the management strategies reported to assist with return to employment?

- What interventions have been described or investigated specifically for addressing work capacity after burn injury?

- What are the models of care used?

- What are the work outcomes achieved?

\section{Stage 2: identifying relevant studies}

Inclusion criteria

We used the Population, Concept and Context Mnemonic to develop and refine the review inclusion criteria. ${ }^{43}$

\section{Population}

Studies that examined adults who are 18-65 years old at the time of their burn injury will be included. Only people with burn injuries that result in cutaneous damage as a result of thermal, chemical, electrical or friction causes will be included as these types of burn injuries are treated by multidisciplinary burn teams in specialist burn services. Burns to internal organs are usually managed by other medical specialities (eg, ophthalmology for ocular burns, respiratory physicians for inhalation burns) and will thus be excluded. Extravasation injuries are also excluded. As cancer treatment can confound return to employment, people with ionising radiation burns from cancer treatment are also excluded. There is no limitation on the physical geolocation of where the burn injury occurred. Both work-related and non-work-related injuries will be considered as long as the participants are working for an income at the time of burn injury.

\section{Concept}

While return to work has been used to describe both paid and unpaid work activities, ${ }^{19} 31$ employment is the means through which people are socially productive and refers to work done specifically to earn money. ${ }^{32}$ Employment undertaken specifically to earn an income for sustenance is defined as work in this review. Papers will be excluded if only unpaid work is investigated. As there are no established definitions of what constitutes return to work in the literature, all potential aspects of resumption of work in regard to capacity (eg, full time, part time), intensity (eg, same duties, reduced duties, work hours), frequency (eg, work days), type (eg, same occupation, different job) or location (eg, same or different employer) of employment are included to consider all employment outcomes. Proxy measures such as sick days, work absence and vocational retraining/training days will also be included.
While participants must have been reported to have had time off from employment because of their burn injury, there are no limits placed on the commencement of time off work or duration of absence.

\section{Context}

Globally, differences in burn injury aetiology, epidemiology and social and healthcare systems in countries can be attributed to age and income status. ${ }^{1}$ There are also differences in safety precautions in the general community, culturally determined behaviours and values regarding employment. It is likely that these differences will affect how return to employment is managed after burn injury. As such, only research reporting on highincome countries (as defined by the World Bank, 2019) will be included. Studies in all healthcare and research settings will be included: hospital-based, communitybased, primary care, specialist care, workplace, nonworkplace and private or public settings.

\section{Stage 3: study selection \\ Search strategy}

The search strategy was designed and extensively tested by the lead author (AK) with the assistance of two research librarians. A three-step search strategy was used: Initial search terms were tested in different combinations in a limited search on Medline and Embase (Ovid platforms) followed by analysis of the title and abstract text and index terms used to describe the articles. To ensure a comprehensive search, the broadest possible search terms were chosen based on return to work as the primary outcome and/or process measure. ${ }^{44} \mathrm{~A}$ second search using all the identified keywords and index terms was then undertaken across all the included databases. The research librarians independently reviewed the search strategy to guide testing. At each iteration, the search strategy was refined after discussion with the research team. The search strategy used for Medline is included in online supplemental appendix 1. Key authors known to publish in this area will also be identified and contacted for further information, if available and willing. Finally, the reference lists of papers included in the full-text retrieval will also be searched for additional studies. Only Englishlanguage documents or English-language abstracts of non-English documents will be included, as the review team do not have capacity for translation.

\section{Information sources}

To ensure a comprehensive search as required for a scoping review, all selected databases (OvidSP: Medline, Embase, PsycINFO, PubMed and Cochrane Central Register of Controlled Trials and EBSCOhost: CINAHL and Scopus) were searched from 2000 to March 2019 and no limits were placed on study design. All types of interventions across all settings and all types of outcome measures will be included. While there are no restrictions on study design, methodology or reporting formats, papers must provide original data for relevant information to 
Table 1 Study selection criteria

Inclusion criteria
The literature on burn injury and return to work in
working adults (aged 18-65 years) who were in paid
employment prior to burn injury

Burn injuries can occur at any setting (eg, home, community, farm, road, waterway, retail/commercial, industrial, construction) and while doing any

activities (ie, non-work, work, leisure, etc)

\section{Examine all employment outcomes \\ - Directly related to return to work \\ - Includes some measurement of employment regardless of specificity}

Examines physical, psychological, social and/or vocational factors that may have a direct effect on ability to work

\section{All study designs, methodologies and reporting formats that present original data and analysed that data}

Research based on populations from high-income countries (World Bank's definition, 2019)

\section{Exclusion criteria}

Burn injuries occurring to internal parts of the body (eg, ocular, lung, oesophageal) without cutaneous involvement; extravasation injuries or ionising radiation burns from cancer treatment

Participant group with burn injury not separately analysed if part of general trauma study

Unpaid work such as volunteer, homemaker and retiree positions

Animal-based, cellular studies, surgical techniques, wound management, burn injury prevention or minimisation, prehospital/intensive care/acute management, burn care staffing issues and event-based reports that do not directly relate to employment after burn injury

Management strategies, interventions and models of care for a comorbidity that is not directly related to a person's ability to work regardless of whether the burn injury was present or not

Commentary/opinion/editorial papers, conference abstracts/theses papers and books/book chapters be extracted. Opinion/editorial papers and conference abstracts will be excluded. For relevant review articles, we will examine individual studies that were included in that review if these meet the scoping review study criteria. The selection criteria presented in table 1 may be further refined as part of the iterative review process to meet the stated research objective.

\section{Selecting the evidence}

Citations will be imported into a reference manager software program (Endnote X9, Clarivate Analytics, Pennsylvania, USA) to remove duplicates. The remaining citations will then be exported to an online screening tool (Rayyan, Qatar Computing Research Institute, Doha, Qatar). ${ }^{45}$ The research team will screen 10 random titles independently using the a priori inclusion criteria to further refine the study criteria. All reviewers will be paired with the first author (who will screen all titles) to independently screen title and abstracts for another 100 records using the refined study criteria to increase consistency among reviewers. Once the calibration exercise is completed, two reviewers (including the first author) will screen the titles and abstracts. Disagreements on study selection will be based on consensus and discussion with other reviewers if required. Results of how selection of sources of evidence was undertaken will be reported using a flow diagram.

\section{Stage 4: charting the data}

A predefined charting form based on the research questions and objectives will be used to extract the data from the selected articles. Two reviewers will trial the extraction in a calibration exercise using the first $10 \%$ of full-text articles retrieved to ensure reliability and accuracy for recording key information. We anticipate changes to the charting form based on the iterative process of a scoping review and will update changes based on consensus between both reviewers. A third reviewer will resolve any disagreements. Key changes made to the charting form will be reported in the final report. Key information to be extracted will include:

- Citation details (author, year and country of origin).

- Study details (study purpose, type of study design, country location, healthcare settings, patient demographics, methodology, interventions, assessment and outcome measures used, methods of analysis, study limitations).

- Conceptual details used (definitions of work, quantification of return to work, classifications of work).

- Key outcomes on return to employment.

\section{Stage 5: collating, summarising and reporting the results}

We plan to identify key themes, methods of assessing outcomes, intervention types and care settings. A biopsychosocial approach is preferable to the current biomedical model of burn injury management when investigating return to employment as it integrates the biological, physical, psychological and social dimensions involved and prevents a narrow focus on singular factors that have predominated previous research in the area of return to work. ${ }^{464}$ It is anticipated that the ICF will provide a useful framework to analyse the findings in relation to participation, which is affected by other components of the ICF such as activity levels, body systems and structures and environmental barriers and facilitators. $^{2}$ However, the ICF does not include classifications for personal factors that are equally important. ${ }^{348} 49$ Personal factors such as motivation, coping and resilience are 
emerging as important to understand burn recovery. ${ }^{31} 5051$ Motivational factors such as decisional balance, self-efficacy and change processes have also been identified as key influences on return to work more broadly. ${ }^{5253}$ We will therefore consider a behavioural change model (such as the Readiness for Change Model) $)^{5254}$ to examine personal motivation factors for returning to employment if appropriate. This will enable a more person-centred approach contextualised to its appropriate sociocultural setting and environment. ${ }^{55}$

The Preferred Reporting Items for Systematic Reviews and Meta-Analyses Extension for Scoping Reviews checklist ${ }^{56}$ will be used to report findings. The reporting format will be descriptive and no critical appraisal will be made of included articles as the purpose of this study is not a systematic review on the quality or rigour of the included research. ${ }^{43}$ Summary tables will be used to present the results. Key themes will be presented in diagrammatically and/or narrative format. We plan to publish the study results and report this in a peer-reviewed journal. Findings will also be presented at conferences.

\section{Stage 6: ongoing consultation of information scientists, librarian and/or experts}

We will engage in ongoing consultation with subject matter experts (on burn injury rehabilitation, vocational rehabilitation and academic librarians) throughout the study to refine the study criteria to align with the stated research questions, aims and objectives.

\section{Patient and public involvement}

The first author's clinical experience with patients and interacting with consumers confirmed the need for a review of return to work for people with burn injuries. This scoping review protocol did not require patient or general public involvement.

\section{DISCUSSION}

This scoping review protocol has been designed to include all relevant information sources to meet the stated study objectives. It is recognised that there could be a high prevalence of mental disorders either pre burn ${ }^{25} 57-61$ or post burn ${ }^{62} 63$ that affect general recovery, ${ }^{64}$ quality of life ${ }^{58}$ and return to work. ${ }^{28}{ }^{65}$ Likewise, alcohol and substance misuse, physical impairments and job-related factors may also contribute to working or not working after injury. ${ }^{39266-69}$ The search strategy was refined and kept broadly focused on return to work so that the search terms used did not inadvertently exclude results ${ }^{43}$ or introduce bias in the search elements. ${ }^{44}$ The use of a biopsychosocial framework to analyse results will enable a comprehensive approach towards recognising the value of return to work in activity participation, reintegration into community and resumption of life roles after burn injury. ${ }^{70}$ It is anticipated that the literature will cover a wide range of areas related to burn injury recovery and work participation. This will likely require a lengthy period of screening as a large volume of citations are expected to be retrieved. Lastly, although this review is focused on high-income countries to reduce heterogeneity in the findings, the inclusion of low-income and middle-income countries is suggested for future research.

\section{Ethics and dissemination}

Ethics approval is generally not required for scoping reviews, as existing literature will be examined. While there is research evidence available in this area of burn recovery, the extent and nature of our knowledge is yet to be determined. Sources of evidence may raise ethical issues, which will be discussed in the findings. This proposed review will be beneficial to burn care and rehabilitation clinicians, researchers and policy developers to plan and manage recovery for individuals with burn injuries who wish to return to employment. Results of the scoping review will be published in a peer-reviewed journal and presented at conferences.

\section{Author affiliations}

${ }^{1}$ Discipline of Occupational Therapy, Faculty of Medicine and Health, The University of Sydney, Sydney, New South Wales, Australia

${ }^{2}$ Department of Occupational Therapy, Royal North Shore Hospital, St Leonards, New South Wales, Australia

${ }^{3}$ Centre for Children's Burns and Trauma Research, University of Queensland, South Brisbane, Queensland, Australia

${ }^{4}$ Discipline of Physiotherapy, Faculty of Medicine and Health, The University of Sydney, Sydney, New South Wales, Australia

${ }^{5}$ Kolling Institute, Northern Sydney Local Health District, St Leonards, New South Wales, Australia

Twitter Zephanie Tyack @tyack_z

Acknowledgements The authors acknowledge Elaine Tam and Yulia Ulyannikova, academic liaison librarians at The University of Sydney, for their technical assistance with developing the initial search terms for this scoping review. AK would like to acknowledge the initial screening and database use training by Bonnie Cheng, hospital librarian at the Douglas Piper Library, Royal North Shore Hospital.

Contributors All authors have made substantial intellectual contributions and conceptualised, drafted, critically appraised and edited the protocol. AK developed the initial protocol manuscript and developed and executed the search strategy as part of her PhD candidature. LM, ZT, MM and JME guided the protocol development and provided major input into the study methodology. All authors provided specific content expertise to inform the study methodology (burn injury, occupational therapy, physiotherapy and return to work).

Funding This work was partly supported by a joint University of Sydney and Northern Sydney Local Health District Allied Health Kickstarter Grant. Opinions and conclusions expressed are those of the authors and area not attributed to the funding bodies. JME is supported by the National Institute of Child Health and Human Development/National Centre for Medical Rehabilitation Research under award number R01HD079076. The content is solely the responsibility of the authors and does not necessarily represent the official views of the National Institutes of Health.

Competing interests None declared.

Patient consent for publication Not required.

Provenance and peer review Not commissioned; externally peer reviewed.

Supplemental material This content has been supplied by the author(s). It has not been vetted by BMJ Publishing Group Limited (BMJ) and may not have been peer-reviewed. Any opinions or recommendations discussed are solely those of the author(s) and are not endorsed by BMJ. BMJ disclaims all liability and responsibility arising from any reliance placed on the content. Where the content includes any translated material, BMJ does not warrant the accuracy and reliability of the translations (including but not limited to local regulations, clinical guidelines, terminology, drug names and drug dosages), and is not responsible for any error and/or omissions arising from translation and adaptation or otherwise.

Open access This is an open access article distributed in accordance with the Creative Commons Attribution Non Commercial (CC BY-NC 4.0) license, which 
permits others to distribute, remix, adapt, build upon this work non-commercially, and license their derivative works on different terms, provided the original work is properly cited, appropriate credit is given, any changes made indicated, and the use is non-commercial. See: http://creativecommons.org/licenses/by-nc/4.0/.

\section{ORCID iDs}

Akane Katsu http://orcid.org/0000-0002-1918-4634

Zephanie Tyack http://orcid.org/0000-0003-3376-5731

\section{REFERENCES}

1 World Health Organisation. A WHO plan for burn prevention and care. Geneva, Switzerland: WHO, 2008.

2 World Health Organisation. International classification of functioning, disability and health. ICF, Geneva: WHO, 2001: 576

3 Wasiak J, McMahon M, Danilla S, et al. Measuring common outcome measures and their concepts using the International classification of functioning, disability and health (ICF) in adults with burn injury: a systematic review. Burns 2011;37:913-24.

4 Hundeshagen G, Suman OE, Branski LK. Rehabilitation in the acute versus outpatient setting. Clin Plast Surg 2017;44:729-35.

5 Jacobson K, Fletchall S, Dodd H, et al. Current concepts burn rehabilitation, part I: care during hospitalization. Clin Plast Surg 2017;44:703-12.

6 Holavanahalli RK, Helm PA, Kowalske KJ. Long-Term outcomes in patients surviving large burns: the musculoskeletal system. J Burn Care Res 2016;37:243-54

7 Ryan CM, Parry I, Richard R. Functional outcomes following burn injury. J Burn Care Res 2017;38:e614-7.

8 Wasiak J, Paul E, Lee SJ, et al. Patterns of recovery over 12 months following a burn injury in Australia. Injury 2014;45:1459-64.

9 Young AW, Dewey WS, King BT. Rehabilitation of burn injuries: an update. Phys Med Rehabil Clin N Am 2019;30:111-32.

10 Attoe C, Pounds-Cornish E. Psychosocial adjustment following burns: an integrative literature review. Burns 2015;41:1375-84.

11 Druery M, Brown TLH, La H Brown T, et al. Long term functional outcomes and quality of life following severe burn injury. Burns 2005;31:692-5.

12 Wiechman SA, McMullen K, Carrougher GJ, et al. Reasons for distress among burn survivors at 6,12 , and 24 months Postdischarge: a burn injury model system investigation. Arch Phys Med Rehabil 2018;99:1311-7.

13 Brych SB, Engrav LH, Rivara FP, et al. Time off work and return to work rates after burns: systematic review of the literature and a large two-center series. J Burn Care Rehabil 2001;22:401-5.

14 Peden M, McGee K, Sharma G. The injury chart book: a graphical overview of the global burden of injuries. Geneva: World Health Organisation, 2002.

15 Pointer S, Tovell A, AlHW. Hospitalised burn injuries, Australia, 201314. Injury research and statistics series No. 102. cat. No. INJCAT 178. Canberra: Australian Institute of Health and Welfare, 2016.

16 Hop MJ, Polinder S, van der Vlies $\mathrm{CH}$, et al. Costs of burn care: a systematic review. Wound Repair Regen 2014;22:436-50.

17 Australian Institute of Health and Welfare. Disease expenditure in Australia. AlHW: Canberra, 2019.

18 Ahn CS, Maitz PKM. The true cost of burn. Burns 2012;38:967-74.

19 Mason ST, Esselman P, Fraser R, et al. Return to work after burn injury: a systematic review. J Burn Care Res 2012;33:101-9.

20 Stergiou-Kita M, Grigorovich A. Guidelines for vocational evaluation following burns: integrated review of relevant process and factors. J Occup Rehabil 2013;23:476-503.

21 Young AE, Roessler RT, Wasiak R, et al. A developmental conceptualization of return to work. J Occup Rehabil 2005; 15:557-68.

22 Yohannan SK, Ronda-Velez Y, Henriquez DA, et al. Burn survivors' perceptions of rehabilitation. Burns 2012;38:1151-6.

23 Carrougher GJ, Brych SB, Pham TN, et al. An intervention bundle to facilitate return to work for Burn-Injured workers: report from a burn model system investigation. J Burn Care Res 2017;38:e70-8.

24 Esselman PC, Askay SW, Carrougher GJ, et al. Barriers to return to work after burn injuries. Arch Phys Med Rehabil 2007;88:S50-6.

25 Fauerbach JA, Engrav L, Kowalske K, et al. Barriers to employment among working-aged patients with major burn injury. J Burn Care Rehabil 2001;22:26-34.

26 Goei $\mathrm{H}$, Hop MJ, van der Vlies $\mathrm{CH}$, et al. Return to work after specialised burn care: a two-year prospective follow-up study of the prevalence, predictors and related costs. Injury 2016;47:1975-82.

27 Helm PA, Walker SC. Return to work after burn injury. J Burn Care Rehabil 1992;13:53-7.
28 Öster C, Ekselius L. Return to work after burn--a prospective study. Burns 2011;37:1117-24.

29 Quinn T, Wasiak J, Cleland H. An examination of factors that affect return to work following burns: a systematic review of the literature. Burns 2010;36:1021-6.

30 Wiechman SA, Carrougher GJ, Esselman PC, et al. An expanded delivery model for outpatient burn rehabilitation. J Burn Care Res 2015;36:14-22

31 Öster C, Kildal M, Ekselius L. Return to work after burn injury: BurnInjured Individuals' perception of barriers and facilitators. Journal of Burn Care \& Research 2010;31:540-50.

32 "employment, n.". Oxford English dictionary online. Oxford University Press, 2020.

33 Wasiak R, Young AE, Roessler RT, et al. Measuring return to work. $J$ Occup Rehabil 2007;17:766-81.

34 Esselman PC. Burn rehabilitation: an overview. . Archives of Physical Medicine and Rehabilitation, 2007: 88(12 SUPPL. 2). S3-6.

35 Peterson J, Pearce PF, Ferguson LA, et al. Understanding scoping reviews: definition, purpose, and process. J Am Assoc Nurse Pract 2017:29:12-16.

36 Conyers LM. A client-focused considering work model for people with emerging or episodic illnesses. J Vocat Rehabil 2018;48:331-43.

37 Iles RA, Sheehan LR, Gosling CM. Assessment of a new tool to improve case manager identification of delayed return to work in the first two weeks of a workers' compensation claim. Clin Rehabil 2020;34:656-66.

38 Jensen AGC. A two-year follow-up on a program theory of return to work intervention. Work 2013;44:165-75.

39 Street TD, Lacey SJ. A systematic review of studies identifying predictors of poor return to work outcomes following workplace injury. Work 2015;51): :373-81.

40 Arksey H, O'Malley L. Scoping studies: towards a methodological framework. Int J Soc Res Methodol 2005;8:19-32.

41 Levac D, Colquhoun H, O'Brien KK. Scoping studies: advancing the methodology. Implementation Science 2010;5:1-9.

42 Peters MDJ, Godfrey CM, Khalil H, et al. Guidance for conducting systematic scoping reviews. Int J Evid Based Healthc 2015;13:141-6.

43 Peters MDJet al. Chapter 11: Scoping Reviews. In A.E, M.Z, eds. In: Joanna Briggs Institute Reviewer's Manual. The Joanna Briggs Institute, 2017: 1-43.

44 Bramer WM, de Jonge GB, Rethlefsen $\mathrm{ML}$, et al. A systematic approach to searching: an efficient and complete method to develop literature searches. J Med Libr Assoc 2018;106:531-41.

45 Ouzzani Met al. Rayyan - a web and mobile APP for systematic reviews systematic reviews 2016;5:210.

46 Cerniauskaite M, Quintas R, Boldt C, et al. Systematic literature review on ICF from 2001 to 2009: its use, implementation and operationalisation. Disabil Rehabil 2011;33:281-309.

47 Escorpizo R, Reneman MF, Ekholm J, et al. A conceptual definition of vocational rehabilitation based on the ICF: building a shared global model. J Occup Rehabil 2011;21:126-33.

48 Momsen AHet al. International Classification of Functioning, Disability and Health in Vocational Rehabilitation: A Scoping Review of the State of the Field. Journal of Occupational Rehabilitation 2018:1-33.

49 Mclntyre A, Tempest S, forward Tsteps. Two steps forward, one step back? A commentary on the disease-specific core sets of the International classification of functioning, disability and health (ICF). Disabil Rehabil 2007;29:1475-9.

50 Bosmans MWG, Hofland HW, De Jong AE, et al. Coping with burns: the role of coping self-efficacy in the recovery from traumatic stress following burn injuries. J Behav Med 2015;38:642-51.

51 Fauerbach JA, Lawrence JW, Fogel J, et al. Approach-avoidance coping conflict in a sample of burn patients at risk for posttraumatic stress disorder. Depress Anxiety 2009;26:838-50.

52 Franche R-L, Krause N. Readiness for return to work following injury or illness: conceptualizing the interpersonal impact of health care, workplace, and insurance factors. J Occup Rehabil 2002;12:233-56.

53 Park J, Roberts MR, Esmail S, et al. Validation of the readiness for return-to-work scale in outpatient occupational rehabilitation in Canada. J Occup Rehabil 2018;28:332-45.

54 Prochaska JO, DiClemente CC. Stages and processes of self-change of smoking: toward an integrative model of change. J Consult Clin Psychol 1983;51:390-5.

55 White C, Green RA, Ferguson S, et al. The influence of social support and social integration factors on return to work outcomes for individuals with work-related injuries: a systematic review. J Occup Rehabil 2019;29:636-59.

56 Tricco AC, Lillie E, Zarin W, et al. PRISMA extension for scoping reviews (PRISMA-ScR): checklist and explanation. Ann Intern Med 2018;169:467-73. 
57 Dyster-Aas J, Willebrand M, Wikehult B, et al. Major depression and posttraumatic stress disorder symptoms following severe burn injury in relation to lifetime psychiatric morbidity. $J$ Trauma 2008;64:1349-56.

58 Low AJF, Dyster-Aas J, Willebrand M, et al. Psychiatric morbidity predicts perceived burn-specific health 1 year after a burn. Gen Hosp Psychiatry 2012;34:146-52.

59 Palmu R, Partonen T, Suominen K, et al. Health-Related quality of life 6 months after burns among hospitalized patients: predictive importance of mental disorders and burn severity. Burns 2015;41:742-8.

60 Palmu R, Partonen T, Suominen K, et al. Functioning, disability, and social adaptation six months after burn injury. J Burn Care Res 2016;37:e234-43.

61 Palmu R, Suominen K, Vuola J, et al. Mental disorders among acute burn patients. Burns 2010;36:1072-9.

62 Anderson NJ, Bonauto DK, Adams D. Psychiatric diagnoses after hospitalization with work-related burn injuries in Washington state. $J$ Burn Care Res 2011;32:369-78.

63 Corry NH, Klick B, Fauerbach JA. Posttraumatic stress disorder and pain impact functioning and disability after major burn injury. J Burn Care Res 2010;31:13-25.
64 Martin L, Byrnes M, Bulsara MK, et al. Quality of life and posttraumatic growth after adult burn: a prospective, longitudinal study. Burns 2017;43:1400-10.

65 Palmu R, Partonen T, Suominen K, et al. Return to work six months after burn: a prospective study at the Helsinki burn center. Burns 2015;41:1152-60.

66 MacKenzie EJ, Morris JA, Jurkovich GJ, et al. Return to work following injury: the role of economic, social, and job-related factors. Am J Public Health 1998;88:1630-7.

67 Anwar MU, Majumder S, Austin O, et al. Smoking, substance abuse, psychiatric history, and burns: trends in adult patients. J Burn Care Rehabil 2005;26:493-501.

68 Kramer CB, Gibran NS, Heimbach DM, et al. Assault and substance abuse characterize burn injuries in homeless patients. J Burn Care Res 2008;29:461-7.

69 O'Brien KH, Lushin V. Examining the impact of psychological factors on hospital length of stay for burn survivors: a systematic review. $J$ Burn Care Res 2019:40:12-20.

70 Schultz IZ, Chlebak CM, Stewart AMSchultz IZ, Gatchel RJ, eds. Impairment, disability, and return to work, in Handbook of return to work. New York: Springer Science+Business Media, 2016: 3-26. 